\title{
Simulated patient deterioration situations reveal taxonomy of the decisions made by nursing students
}

Citation: Bucknall TK, Forbes H, Phillips NM, et al. An analysis of nursing students' decisionmaking in teams during simulations of acute patient deterioration. J Adv Nurs. 2016 Oct;72(10):2482-94.

\section{Commentary \\ Implications for practice and research}

- This study provides a taxonomy of the decisions made by nursing students in patient deterioration simulations that can clarify the expected outcomes of decision-making education.

- The results highlight the influence of knowledge and non-technical skills on student decision-making in a team-based context.

- Further research is needed to examine the generalizability of this taxonomy and evaluate the effectiveness of different approaches to teaching decision-making.

\section{Context}

Simulation is increasingly used to prepare nursing students to recognize and respond to patient deterioration and research suggests its effectiveness to improve students' decision-making ability. ${ }^{1}$ However, the best approaches to simulation for achieving this outcome are less understood. This study by Bucknall and colleagues described nursing students' decision-making in team-based patient deterioration simulations.

\section{Methods}

The purpose of this qualitative exploratory study was to describe the types of decisions made by third-year nursing students during simulations of patient deterioration as well as the factors influencing their decisions and the sources of information they use. Twelve teams of two to three students participated in three different 8-minute simulations of cardiac problems, shock, and respiratory difficulties involving a 70-year-old male standardized patient. After the simulations, students reviewed videos of their performance and were asked to recall and explain their decision-making.

\section{Findings}

Students gathered information from three sources to understand what was happening in each scenario: observation of patient's behavior, problem-focused assessment, and chart review. Decisions that students made were grouped by the researchers into 11 categories: information seeking; patient assessment; diagnostic; intervention/treatment; evaluation; escalation; prediction; planning; collaboration; communication and reflective. Patient distress, students' lack of knowledge, absence of experienced staff, and unfamiliarity with the patient, the chart, and the equipment were found to have negative influences on student decision-making.

The study revealed that information seeking and patient assessment decisions were narrowly focused and often revolved around collection of a single vital sign related to patient's main presenting symptom. Decisions to intervene relied on diagnoses of problems that were often based on a single assessment cue. Students who identified a condition in one scenario were quick to recognize it in subsequent simulations.

Results also identified strong influences of interpersonal skills on nursing students' decisionmaking. Exploration of collaboration and communication decisions revealed that many students tried to solve problems on their own and asked others only when they had exhausted their 
knowledge. Communication with the patient was found to be minimal and directive. Few students articulated plans or predictions in a manner suggesting they were anticipating how the simulations could unfold.

\section{Commentary}

This was a descriptive study of decisions by nursing students in patient deterioration simulations. Students appeared vulnerable to cognitive biases, ${ }^{2}$ such as fixation on a single piece of data and reliance on recent examples when approaching new situations. The responses of the students to the simulations did not reflect high levels of teamwork, leadership, communication, or other nontechnical skills important to patient safety. ${ }^{3}$ Taken together, the findings were consistent with the existing body of literature on healthcare professional decision-making.

The results provide insights into the challenges nursing students face when confronted with patient deterioration and offer guidance for setting more precise goals for educational strategies. This study also raises important questions about the effectiveness of current educational strategies in terms of fostering decision-making and addressing common biases and errors. While existing evidence does not support the effectiveness of training for the recognition of cognitive biases, ${ }^{4}$ there remains a need for educators to be aware of those possible biases and to help students avoid these pitfalls in clinical reasoning. Moreover, the study highlights the critical importance of targeting the development of interpersonal skills in designing educational strategies to improve student decision-making and patient safety.

Future research should validate the taxonomy in other settings and examine the extent to which the decisions are elicited by patient safety scenarios beyond patient deterioration simulations. It appears necessary to determine if this taxonomy applies to students at different levels in their training, as well as practicing nurses at different levels of experience. If validated, this taxonomy could serve as a framework for the development and evaluation of instructional strategies and stimulate new research in this field.

\section{Commentator details}

Name: Patrick Lavoie

Affiliation: William F. Connell School of Nursing, Boston College

Correspondence address: Boston College, William F. Connell School of Nursing, Maloney Hall, 140 Commonwealth Ave, Chestnut Hill MA 02467

Email: Patrick.lavoie@bc.edu

\section{References}

1. Fisher D, King L. An integrative literature review on preparing nursing students through simulation to recognize and respond to the deteriorating patient. $J$ Adv Nurs 2013,69;2375-88

2. Blumenthal-Barby JS, Krieger H. Cognitive biases and heuristics in medical decision making: a critical review using a systematic search strategy. Med Decis Making 2015,35;539-57.

3. Gordon M, Darbyshire D, Baker P. Non-technical skills training to enhance patient safety: a systematic review. Med Educ 2012,46;1042-54.

4. Norman GR, Monteiro SD, Sherbino J, Ilgen JS, Schmidt HG, Mamede S. The causes of errors in clinical reasoning: Cognitive biases, knowledge deficits, and dual process thinking. Acad Med 2016,92;23-30.

Competing interests: None 